\title{
Chronic myeloid leukaemia in the South African public health setting: Are we reaching the European LeukemiaNet targets for frontline therapy?
}

\begin{abstract}
Authors:
Tshifhiwa B. Sikhipha ${ }^{1}$ (])

Gina Joubert ${ }^{2}$ (I)

Claire L. Barrett ${ }^{1} \bullet$

Christopher D. Viljoen ${ }^{3}$ (D)

Shivani Dhar ${ }^{1}$ (I)

Jacques L.R. Malherbe ${ }^{1}$ (I)

Affiliations:

${ }^{1}$ Department of Internal

Medicine, Faculty of Health

Sciences, University of the

Free State, Bloemfontein,

South Africa

${ }^{2}$ Department of Biostatistics, Faculty of Health Sciences, University of the Free State, Bloemfontein, South Africa

${ }^{3}$ Department of Haematology and Cell Biology, Faculty of Health Sciences, University of the Free State, Bloemfontein, South Africa
\end{abstract}

Corresponding author: Jacques Malherbe malherbejlr@ufs.ac.za

Dates:

Received: 08 Oct. 2020

Accepted: 10 Nov. 2020

Published: 09 Dec. 2020

How to cite this article: Sikhipha TB, Joubert G, Barrett CL, Viljoen CD, Dhar S, Malherbe JLR. Chronic myeloid leukaemia in the South African public health setting: Are we reaching the European LeukemiaNet targets for frontline therapy? S. Afr. j. oncol. 2020;4(0), a158. https://doi. org/10.4102/sajo.v4i0.158

\section{Copyright:}

(C) 2020. The Authors.

Licensee: AOSIS. This work is licensed under the

Creative Commons

Attribution License.

Read online:
Background: Successful therapy of chronic myeloid leukaemia (CML) relies on close monitoring of patients' response to therapy using the standardised real-time quantitative polymerase chain reaction (RQ-PCR) or fluorescent in situ hybridisation (FISH) at 3, 6 and 12 months. The European LeukemiaNet has published recommended therapeutic targets for frontline therapy at specific time points. Imatinib, a tyrosine kinase inhibitor, is used for the frontline treatment of chronic and accelerated phase CML in our setting.

Aim: The aim of this study was to evaluate treatment response of patients with CML in chronic or accelerated phase treated with imatinib during the first 2 years of therapy.

Setting: Universitas Academic Hospital, South Africa.

Methods: In this analytical cohort study, a retrospective file review of all chronic and accelerated phase CML diagnosed between 2009 and 2016, who were initiated on imatinib as front-line therapy, was performed. Clinical and laboratory data were collected for different time intervals as recommended by European LeukemiaNet guidelines.

Results: Thirty-seven patients met the inclusion criteria. An optimal response was obtained in $82.6 \%, 54.2 \%, 50.0 \%$ and $66.7 \%$ of patients at $3,6,12$ and 18 months, respectively.

Conclusion: The patient outcomes were comparable with other published studies, with two-thirds of patients achieving an optimal response at 18 months. It is important, however, that factors contributing to suboptimal responses in the remaining third of patients should be explored.

Keywords: chronic myeloid leukemia; CML; outcomes; frontline therapy; tyrosine kinase inhibitors; imatinib.

\section{Introduction}

Chronic myeloid leukaemia (CML) is a myeloproliferative neoplasm caused by a translocation between chromosome 9 and 22, denoted as $\mathrm{t}(9 ; 22)(\mathrm{q} 34 ; \mathrm{q} 11.2)$, involving the fusion of the breakpoint cluster region (BCR) gene on chromosome 22q11.2 and the Abelson gene (ABL1) on chromosome $9 \mathrm{q} 34$. This translocation is known as the Philadelphia chromosome. ${ }^{1,2}$ The resultant fusion oncogene, $B C R-A B L 1$, encodes a tyrosine kinase that drives clonal proliferation of haematopoietic stem cells and mainly affects the granulocytic lineage. ${ }^{1,2}$ Tyrosine kinase inhibitors (TKI) target and suppress the BCR-ABL1 tyrosine kinase and have revolutionised the outcome of CML. Imatinib was the first available TKI, with nilotinib and dasatinib developed later as more potent second-generation agents. ${ }^{3,4}$

Treatment response to TKIs is monitored by fluorescence in situ hybridisation (FISH) or real-time quantitative reverse transcriptase polymerase chain reaction (RQ-PCR), and specific guidelines have been published on the targets of therapy. 5,6 Successful therapy with a TKI relies on close monitoring of the patient's response by means of the standardised RQ-PCR or cytogenetic investigations at 3, 6 and 12 months, according to the guidelines proposed by the European LeukemiaNet (ELN). ${ }^{5}$ Despite the development of various scoring systems such as the Sokal, European Treatment and Outcome Study (EUTOS) and the Eutos long-term survival score (ELTS), the most important prognostic factor is a response to therapy with a TKI. ${ }^{5,7,8}$ Patients achieving therapeutic targets can expect prolonged survival approaching that of the normal age-matched population. ${ }^{3,49}$

Imatinib is the only frontline therapy available for chronic and accelerated phase CML at the Universitas Academic Hospital (UAH) in Bloemfontein, South Africa. There are no published 
data available regarding the achievement of therapeutic targets to TKIs in South Africa or in our setting, and consequently, it is unclear whether we are achieving the treatment targets. To address this paucity of data, a retrospective file review was conducted to describe the response to treatment during the first 2 years of therapy for newly diagnosed CML patients in chronic and accelerated phase at UAH. The study aimed to monitor response to treatment in order to assess whether patients treated at our institution met the treatment targets for CML as defined by the ELN for frontline TKI therapy.

\section{Methods \\ Study design and setting}

A retrospective analytical cohort study was performed. The study was performed at UAH, which is the only tertiary referral hospital in the Free State Province of South Africa. Universitas Academic Hospital (UAH) provides specialist haematology care to parts of the Northern Cape province and a neighbouring country, Lesotho.

\section{Participants}

All chronic or accelerated phase CML patients older than 16 years of age, who were diagnosed between 2009 and 2016 at the UAH Division of Clinical Haematology, were included in the study. Patients who presented in the blast phase and those without follow-up data were excluded. The World Health Organization's (WHO) definition of accelerated and blast phase was used..$^{10}$ Since 2012, the International Scale (IS) was used to report RQ-PCR results at UAH. ${ }^{5}$

\section{Measurement}

Demographic, clinical and laboratory data were collected from patient's files, the hospital electronic patient record system, Meditech (Medical Information Technology, Incorporated) and laboratory information services (National Health Laboratory Services, NHLS-Labtrak, and other laboratory registers). Baseline clinical data, comorbidities, HIV status and spleen size were documented. Laboratory data collected included full blood count, t(9; 22) FISH and/or BCR-ABL1 RQ-PCR results. Data regarding imatinib dose, drug compliance and adverse events were retrieved from patient's files. The Sokal risk score for each patient was calculated from the data available at baseline. The percentage of patients reaching treatment endpoints as defined by the ELN 2013 guidelines ${ }^{5}$ was determined.

\section{Data analysis}

Data were analysed by the department of Biostatistics at the University of Free State. Categorical variables are summarised by frequencies and percentages and numerical variables by medians because of skew distributions. Univariate analyses were done to test the association between major cytogenetic response or major molecular response and prognostic factors, including baseline spleen size, Sokal score, leucocyte count and platelet count.

\section{Routine patient management}

As a standard practice, all patients receive frontline therapy with imatinib. For patients diagnosed in the chronic phase, imatinib $400 \mathrm{mg}$ is prescribed, whilst patients diagnosed in the accelerated phase receive $600 \mathrm{mg}$ imatinib. Escalation of imatinib dose is implemented in the event of suboptimal response to standard dose, and dose reductions are made for toxicities. The site performs FISH or RQ-PCR on the IS every 3 months until two values are below $0.1 \%$, thereafter 6-monthly monitoring is performed. Compliance is assessed when patients do not achieve the expected treatment endpoint according to the treatment protocol. Imatinib is changed to second-line therapy (nilotinib or dasatinib) in the event of adverse effects or treatment failure once compliance has been assured. In our setting, nilotinib is generally used as second-line and dasatinib as third-line agents, unless $B C R-A B L 1$ mutational analysis directs otherwise.

\section{Ethical consideration}

This study was approved by the Health Sciences Research Ethics Committee (HSREC) of the University of the Free State (UFS-HSD2017/0065) and the Free State Province Department of Health. Each patient's file was allocated a study number that was used in the data collection to ascertain the confidentiality of patients' identity.

\section{Results \\ Baseline data}

Thirty-seven patients met the inclusion criteria, of which $31(83.8 \%)$ patients were in the chronic phase and six (16.2\%) in the accelerated phase. All patients were prescribed imatinib as frontline therapy as per treatment recommendation. The dose of imatinib was $400 \mathrm{mg}$ for $32(86.5 \%)$ patients, $600 \mathrm{mg}$ for four $(10.8 \%)$ patients and $800 \mathrm{mg}$ for a single $(2.7 \%)$ patient.

The majority of patients were females $(n=23 ; 62.2 \%)$. The median age of patients was 42.5 years with a range of 16-76 years. The age group 31-50 years represented the largest age group $(n=10 ; 27.0 \%)$, and $27(73.0 \%)$ patients were under the age of 50 years. Comorbidities were noted in $25(67.6 \%)$ patients, with hypertension being the most prevalent comorbidity $(n=12 ; 32.4 \%)$, human immunodeficiency virus (HIV) infection was present in four $(10.8 \%)$ and diabetes mellitus in three $(8.1 \%)$ patients. Other comorbidities were of low frequency and included hypertensive heart disease, stroke, major depressive disorder, previous breast cancer, psoriasis and neural tube defect (one patient each) and asthma (two patients).

Baseline clinical and laboratory data are summarised in Table 1.

\section{Adverse events}

The most frequent adverse events recorded were haematological (anaemia, thrombocytopenia or neutropenia). 
TABLE 1: Clinical and laboratory median values at baseline.

\begin{tabular}{lccc}
\hline Parameter & Number of patients data & Median & Range \\
\hline Spleen size $(\mathrm{cm}) \dagger$ & 36 & 11 & $0-25$ \\
Leucocytes $\left(\times 10^{9} / \mathrm{L}\right)$ & 37 & 201.5 & $29.7-637.0$ \\
Platelets $\left(\times 10^{\%} / \mathrm{L}\right)$ & 37 & 325 & $98-1555$ \\
FISH $(\%)$ & 25 & 70 & $3-99$ \\
BCR-ABL1 (IS) (\%) & 19 & 19 & $0.06-279.69$ \\
Sokal score & 37 & 1.14 & $0.49-4.49$ \\
\hline
\end{tabular}

$\dagger$, Below costal margin; FISH: fluorescence in situ hybridisation; IS: International Scale.

TABLE 2: Response to frontline imatinib at Universitas Academic Hospital.

\begin{tabular}{|c|c|c|c|c|c|c|}
\hline \multirow[t]{2}{*}{ Time } & \multicolumn{2}{|c|}{ Optimal } & \multicolumn{2}{|c|}{ Warning } & \multicolumn{2}{|c|}{ Failure } \\
\hline & $n$ & $\%$ & $n$ & $\%$ & $n$ & $\%$ \\
\hline 3 months $(n=23)$ & 19 & 82.6 & 4 & 17.4 & 0 & 0 \\
\hline 6 months $(n=24)$ & 13 & 54.2 & 7 & 29.2 & 4 & 16.7 \\
\hline 12 months $(n=24)$ & 12 & 50.0 & 8 & 33.3 & 4 & 16.7 \\
\hline 18 months ( $n=24)$ & 16 & 66.7 & 5 & 20.8 & 3 & 12.5 \\
\hline
\end{tabular}

Because of the retrospective nature of this study, adverse events were not graded. Adverse events were documented in $32.4 \%(n=12)$ patients, whilst $10.8 \%(n=4)$ had more than one toxicity. Haematological toxicity was found in $24 \%$ of patients $(n=9)$, with four patients having neutropenia, one patient anaemia, one patient thrombocytopenia and three patients had unspecified cytopenias. These were followed by muscle cramps in $8.1 \%(n=3)$, and nausea, vomiting, diarrhea, headache, skin rash and lower limb oedema, each in $3 \%(n=1)$ of patients.

Poor compliance was documented in the records of $18.9 \%$ $(n=7)$ of patients and $16.2 \%(n=6)$ had missed scheduled clinic dates. Dose adjustments were made where indicated. Dose reductions were adequate to control adverse events in $4(11 \%)$ patients. Imatinib dose was not reduced to below $300 \mathrm{mg}$ daily. One patient's treatment was changed to nilotinib because of severe muscle cramps.

\section{Response}

Although 37 patients qualified for the study, they were not evaluable at each time point $(3,6,12$ and 18 months). Nine patients only had baseline data available, with one documented death and 8 lost to follow up. The remaining 28 patients had response data that were evaluable at some time point after the initial diagnosis, and 17 had data for each time point. The results are reported for all patients with available information at a given time point.

Complete cytogenetic response $(\mathrm{t}(9 ; 22)$ by $\mathrm{FISH}=0 \%$, or $B C R-A B L 1<1 \%)$ was seen in 54.2\% $(n=13 / 24), 83.3 \%$ $(n=20 / 24)$ and $87.5 \%(n=21 / 24)$ at 6,12 and 18 months, respectively. Major molecular response $(B C R-A B L 1<0.1 \%)$ was achieved in $50.0 \%(n=12 / 24)$, and $66.7 \%(n=16 / 24)$ at 12 and 18 months, respectively. Because of small numbers, none of the prognostic factors assessed in the univariate analysis were predictive of response. Response according to the ELN criteria ${ }^{5}$ is summarised in Table 2. At 3 months, an optimal response was noted in 19 of $23(82.6 \%)$ patients, with none failing at this time. A third $(66.7 \%, n=16 / 24)$ maintained optimal response and $12.5 \%(n=3 / 24)$ failed treatment with frontline imatinib at 18 months.

\section{Discussion}

In this study, we investigated the response to treatment with frontline imatinib during the first 2 years of therapy in patients with newly diagnosed CML treated at UAH in the period 2009-2016. To our knowledge, this has been the first study of its kind for patients with CML performed in South Africa.

Our study showed a younger median age at diagnosis (42.5 years) compared to the median age of 50 years reported in other studies. ${ }^{11,12,13}$ Although this finding may be attributed to the small sample size, we have observed that many haematological malignancies are diagnosed at a younger age in our population. Whether this is because of a younger population in general or because of biological differences in susceptibility is unknown.

Treatment response was evaluated according to the ELN guidelines published in 2013..$^{5}$ New guidelines were published in 2020 with minor changes in the definition of failure at 3 months. ${ }^{14}$ As our clinical practice during the study period more closely followed the 2013 guidelines, we evaluated responses against these guidelines. The majority of patients attained an optimal response, which was comparable with a number of international studies. ${ }^{9,15}$

Figure 1 compares imatinib response at UAH and imatinib response according to ELN recommendations as published by Zackova et al. ${ }^{9}$ It should be noted that Zackova included only data from patients in the chronic phase, whilst in this study, data from patients in both the accelerated and chronic phase were included. No significant difference between the two studies was noted at 3 and 18 months ( $p=0.79$ and $p=0.62$, respectively). A significant difference at 6 months $(p=0.01)$ and a close to significant difference at 12 months $(p=0.06)$ were noted. The exclusion of patients in the accelerated phase in Zackova's study may account for these differences. ${ }^{9}$ Furthermore, it has been noted in our setting that patients do not always understand that CML is a chronic disease and that long-term compliance is necessary. The importance of compliance is discussed at each followup visit. This may explain the good response initially, the wane in response, and then the improved response at 18 months.

No clinical or laboratory parameters predictive of response were identified in our study. Although three of the four $(75.0 \%)$ patients who failed treatment at 6 months (as shown in Table 2) had high Sokal scores, we could not draw any conclusions from these results because of the small sample size. However, Druker et al. reported that patients with high-risk scores had lower rates of a complete cytogenic response. ${ }^{16}$

The drug toxicity profile of patients in our study was comparable to published data. ${ }^{15,16}$ Most documented toxicities 


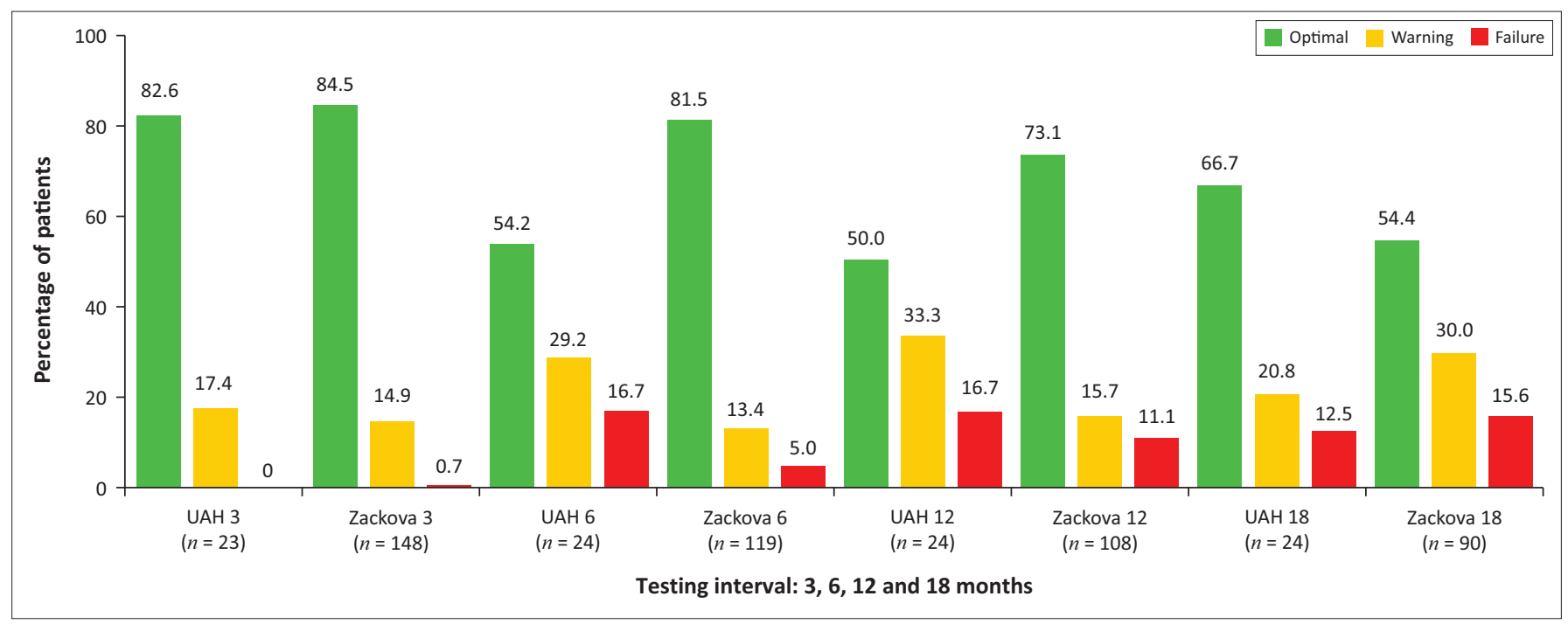

FIGURE 1: Overall response to frontline imatinib, comparing Universitas Academic Hospital data and findings as reported by Zackova et al. ${ }^{9}$

were haematological, which is similar to what has been described by Kantarjian et al. ${ }^{17}$ Although oedema, muscle cramps, diarrhoea, nausea, musculoskeletal pain and skin rash have been described as a common side-effect of imatinib, ${ }^{15}$ these were described in a low frequency in our patients, which might have been attributed to poor record record keeping or under-reporting.

Reasons for poor compliance and the large number of patients with missed clinic dates in the study population were not clearly documented in the patient records. Patients who attend the Haematology Clinic are largely dependent on hospital or public transport. As our clinic serves the Free State, Northern Cape and Lesotho, patients may have to travel up to $950 \mathrm{~km}$ to attend the clinic. Imatinib is stocked at the pharmacy at UAH, whilst all other hospitals in the province have to order it for specific patients, which may cause delays and make prescriptions difficult to fill. Patients from Lesotho experience additional challenges where roads are often in a poor condition and patients may experience delays at border posts. These factors may contribute to missed appointments, but more importantly, these patients are unable to collect imatinib, which is not available in Lesotho.

Additional factors that may influence compliance in our population include poor socio-economic circumstances, informal employment resulting in difficulty to get time off from work to attend clinics, and a lower literacy rate. Furthermore, South Africa is a country with 11 official languages and physicians often are not able to speak the same language as the patient, resulting in consultations being conducted with the assistance of interpreters, which may influence the patient's understanding of their disease and the need for compliance.

Our findings, together with several international studies, emphasise that imatinib is effective in treating CML, but that a significant proportion of patients does fail frontline imatinib. Deeper and earlier responses have been shown using second generation TKIs, but it comes at the expense of more long-term toxicities. The more potent second-generation TKI's, nilotinib and dasatinib, are not freely available as frontline therapy in our setting, and the use of these agents is limited by cost. Where these agents are available in the frontline setting, the choice is generally individualised and based on CML risk score, side-effect profile, comorbidities, patient preference and cost.

Treatment failure in our setting may be because of poor access to uninterrupted treatment or non-compliance. Drug interactions, and interactions with herbal remedies, also play an important role. Mutations in $B C R-A B L 1$ and other molecular mechanisms lead to resistance to therapy in some patients. ${ }^{1,3,5,6}$

The study is limited by the small sample size and retrospective nature of data collection. Some patients also had missing data at different intervals of follow-up.

\section{Conclusion}

This study showed that the majority of patients attained an optimal response by 18 months of treatment, but a significant proportion still failed frontline imatinib. There is a need to identify factors that contribute to treatment failure, such as non-compliance, social circumstances, interrupted drug supply and advanced disease at presentation. Outcomes of CML patients at UAH at 18 months compare favourably with other published real-world experiences.

\section{Acknowledgements}

The authors would like to thank Dr. Daleen Struwig, medical writer and editor, Faculty of Health Sciences, University of the Free State, for technical and editorial preparation of the manuscript. 


\section{Competing interests}

The authors have declared that no competing interest exists.

\section{Authors' contributions}

T.B.S., C.L.B. and J.L.R.M. developed the protocol and wrote the article. C.D.V. and G.J. assisted with protocol development and edited the article. T.B.S. and S.D. collected data. G.J. analysed the data. All authors approved the final version of the document.

\section{Funding information}

This research received no specific grant from any funding agency in the public, commercial or not-for-profit sectors.

\section{Data availability statement}

Data are available from the corresponding author upon reasonable request.

\section{Disclaimer}

The views and opinions expressed in this article are those of the authors and do not necessarily reflect the official policy or position of any affiliated agency of the authors.

\section{References}

1. Baccarani $M$, Dreyling $M$. Chronic myeloid leukaemia: ESMO clinical practice guidelines for diagnosis, treatment and follow up. Ann Oncol. 2010;21(Suppl 5):165-167. https://doi.org/10.1093/annonc/mdq201

2. Faderl S, Talpaz M, Estrov Z, O'Brien S, Kurzrock R, Kantarjian HM. The biology of chronic myeloid leukemia. N Engl J Med. 1999;341(3):164-172. https://doi.
org/10.1056/NEJM199907153410306
3. Cortes J, Kantarjian $\mathrm{H}$. How I treat newly diagnosed chronic phase CML. Blood. 2012;120(7):1390-1397. https://doi.org/10.1182/blood-2012-03-378919

4. O'Brien SG, Guilhot F, Larson RA, et al. Imatinib compared with interferon and low dose cytarabine for newly diagnosed chronic phase myeloid leukemia. N Engl J Med. 2003;348(11):994-1004. https://doi.org/10.1056/NEJMoa022457

5. Baccarani $M$, Deininger MW, Rosti $G$, et al. European LeukemiaNet recommendations for the management of chronic myeloid leukemia: 2013. Blood. 2013;122(6):872-884. https://doi.org/10.1182/blood-2013-05-501569

6. Hughes $T$, Deininger $M$, Hochhaus A, et al. Monitoring CML patients responding to treatment with tyrosine kinase inhibitors: Review and recommendations for harmonizing current methodology for detecting BCR-ABL transcripts and kinase domain mutations and for expressing results. Blood. 2006;108(1):28-37. https:// doi.org/10.1182/blood-2006-01-0092

7. Sokal JE, Cox EB, Baccarani $M$, et al. Prognostic discrimination in 'good-risk' chronic granulocytic leukemia. Blood. 1984;63(4):789-799. https://doi.org/ 10.1182/blood.V63.4.789.bloodjournal634789

8. Pfirrmann $M$, Baccarani $M$, Saussele $S$, et al. Prognosis of long-term survival considering disease-specific death in patients with chronic myeloid leukemia. Leukemia. 2016;30(1):48-56. https://.doi.org/10.1038/leu.2015.261

9. Zackova D, Klamova H, Dusek L, et al. Imatinib as first line treatment of patients with chronic myeloid leukemia diagnosed in the chronic phase: Can we compare real life data to the results from clinical trials? Am J Hematol. 2011;86(3):318-321. https://doi.org/10.1002/ajh.21942

10. Swerdlow SH, Campo E, Harries NL, et al. WHO classification of tumours of haematopoietic and lymphoid tissues. 4th ed. Geneva: World Health Organization; 2008.

11. Hellenbrecht A. European LeukemiaNet registry [homepage on the Internet]. 2020 [cited 2020 Apr 27]. Available from: https://www.eutos.org/content/home/ index_eng.html.

12. Louw VJ. Chronic myeloid leukemia in South Africa. Hematology. 2012;17(Suppl 1):S75-S78. https://doi.org/10.1179/102453312X13336169155817

13. Louw VJ, Dreosti L, Ruff $P$, et al. Recommendations for the management of adult chronic myeloid leukaemia in South Africa. S Afr Med J [serial online]. 2011 [cited 2020 Apr 27];101(11):840-846. Available from: https://www.samj.org.za/index. $\mathrm{php} / \mathrm{samj} /$ article/view/5223

14. Hochhaus A, Baccarani M, Silver RT, et al. European LeukemiaNet 2020 recommendations for treating chronic myeloid leukemia. Leukemia. 2020;34(4):966-984. https://doi.org/10.1038/s41375-020-0776-2

15. Hughes TP, Branford S, White DL, et al. Impact of early dose intensity on cytogenetic and molecular responses in chronic-phase CML patients receiving 600 $\mathrm{mg} /$ day of imatinib as initial therapy. Blood. 2008;112(10):3965-3973. https:// doi.org/10.1182/blood-2008-06-161737

16. Druker BJ, Guilhot F, O'Brian SG, et al. Five year follow-up of patients receiving imatinib for chronic myeloid leukemia. N Engl J Med. 2006;355(23):2408-2417. https://doi.org/10.1056/NEJMoa062867

17. Kantarjian H, Sawyers C, Hochhaus A, et al. Hematologic and cytogenetic responses to imatinib mesylate in chronic myelogenous leukemia. N Engl J Med. 2002;346(9):645-652. https://doi.org/10.1056/NEJMoa011573 